\title{
Event-frequency judgments as a function of the linguistic frequency and single or paired presentation of target words: I. Task with unique multiple traces
}

\author{
MELVIN H. MARX \\ Georgia State University, Atlanta, Georgia
}

\begin{abstract}
Words that are high or low in linguistic frequency were presented singly or in mixed pairs to two small groups of college-student volunteers. Event frequency was manipulated within list: $0,1,2,3$, and 4 occurrences of words, single or paired. The subjects' task was to compose a brief sentence for each slide, including the word(s) shown; different sentences were requested for repeated words. An unexpected event-frequency test was then given. The main results were that reliably more subjects judged event frequency correctly for words low in linguistic frequency when those words were singly presented, confirming Rao's conclusion (1983); the same words were given numerically higher event-frequency judgments when paired. Implications of the results are discussed, particularly with respect to the earlier demonstrations of superior recall of words low in linguistic frequency compared with recall of words high in linguistic frequency when the two types were presented alternately.
\end{abstract}

Rao (1983) found that words low in linguistic frequency (loLF) were given more accurate event-frequency (EF) estimates than were words high in linguistic frequency (hiLF). On the assumption that EF judgment and recognition memory are based on similar memory mechanisms (Harris, Begg, \& Mitterer, 1980), Rao's result is consistent with the commonly reported superior recognition, but not recall, of loLF words (e.g., McCormack \& Swenson, 1972; Sumby, 1963). However, loLF words were better recalled than more common words when the two types of words were presented in mixed lists (Duncan, 1974; May \& Tryk, 1970). This result was replicated by May, Cuddy, and Norton (1979), whose loLF words were more poorly recalled after homogeneous presentation, equally well recalled after blocked presentation, and better recalled than common words after alternating presentation.

This study was designed to explore the effects of contrasting presentation of words of high and low LF on the EF measure. Superior EF judgments for loLF words in mixed pairs would be expected if such linguistic contrast has the same effects that have been shown for word recall, on the assumption that the retrieval of the multiple memory traces would be improved. The cover task used, the composition of short, unique sentences utilizing the words presented, was designed to maximize the individuality and the recallability of the memory traces.

The data were collected while the author was supported by a Research Career Award from the National Institute of Mental Health to the University of Missouri-Columbia. The author thanks Michael Johnson for making available the student volunteers in his two laboratory sections. Address reprint requests to: Melvin H. Marx, Department of Psychology, Georgia State University, Atlanta, GA 30303.

\section{METHOD}

\section{Subjects}

A total of 24 students in two laboratory sections of introductory psychology volunteered to participate and provided usable training and test records; an additional 2 students participated but did not provide usable records.

\section{Experimental Design}

The subjects were shown a series of single or paired (but unrelated) words. Pairs of words always consisted of one hiLF and one loLF word. Manipulation of EF within the list produced 0, 1, 2, 3, and 4 occurrences of hiLF and loLF words in both the single and the paired format. Advantitious variations in the memorability of the randomly ordered words were controlled for the presentation variable by the reversal of words in the single and the paired formats in the list given the second of the two classes. The EF test was administered without prior notice immediately following the study session.

\section{Word Lists}

There were 30 critical study slides in each list. Of these, 20 presented single words and 10 showed paired words, each of which was a target word, one from the hiLF and the other from the loLF word pool. The hiLF words were from the highest (AA) frequency category in the Thorndike and Lorge (1944) norms, and the loLF words were from very low frequency levels (no more than nine occurrences per million words). The single words, half hiLF and half loLF, were presented $1,2,3$, or 4 times. The EF word with a frequency of 1 (EF1) was necessarily paired with the EF4 word in the other LF category (because that word had to be shown 4 times); the EF2 word was paired with the EF3 and the EF4 words from the other LF set; the EF3 word was paired with each of the EF words in the other set except the EF1 word; and the EF4 word was paired with each of the 4 LF words in the other set. Slide order and position of the 2 words on the slide were randomly determined. The mean number of intervening words between repetition of target words was 10.8 for hiLF and 9.6 for loLF words in the single-word format, and 7.7 for hiLF and 7.9 for loLF words in the paired-words format.

In addition to the 30 critical study slides, there were two primacy and two recency buffers, each with one single word (paint, greed) and one 
pair of words (power-cycle, badge-value). The second list contained the same buffers and the same target words, but the target words were reversed with respect to their single or paired presentation.

All words were five letters in length. The target words in the hiLF set were dream and guess (EF4), earth and shore (EF3), heart and party (EF2), music and table (EF1), and horse and scene (EFO). The loLF target words were clown and flare (EF4), aroma and easel (EF3), decoy and prose (LF2), brawl and oasis (EF1), and alibi and diary (LFO).

The test list of 20 target words was randomly arranged, except that each half of the list had 2 words from each of the EF categories (0-4) and no more than 3 single or paired hiLF or loLF words, making 5 single and 5 paired words overall. Also, no more than 2 consecutive words of the hiLF or the loLF or of the single or the paired format were permitted.

\section{Procedure}

Subjects were tested in two groups at their assigned class times. They were told that their task was to compose short sentences including the word or words shown on each slide and to write them on the answer sheets provided. They were also told that the pairings were random, that some of the words would be repeated but no word pairs would be exactly repeated, and that different sentences were to be composed for repeated words.

The projector was set at a $15-\mathrm{sec}$ presentation rate, and four slides were shown for practice (peace; audit-house; crank; melon-start).

After students completed the task on the 34 slides and the answer sheets were collected, a second set of answer sheets was distributed. Subjects were told that they would now be shown slides with single words and that their task was to "estimate as closely as possible how many times each word occurred during the first part of the experiment." They were told that some of the words had not been shown, so that the lowest numerical score should be zero, but they were not given an upper limit. The projector was set at an $8-\mathrm{sec}$ rate.

\section{RESULTS}

Table 1 shows the proportions of correct EF judgments over the five frequencies for the hiLF and loLF words and for single and paired word presentations. The reliability of the overall advantage of single loLF words over single hiLF words $(.67-.53=.14)$ is suggested by the fact that 14 subjects made more correct EF judgments in that direction, compared with 4 showing the opposite tendency. With six ties divided evenly, this difference varied reliably from an assumed chance $50 \%$ distribution $\left[\chi^{2}(1)\right.$ $=4.17, p<.05]$. (This procedure of splitting ties and assuming a chance $50 \%$ distribution is used in all subsequent chi-square analyses.) The smaller difference in the same direction between paired hiLF and loLF words $(.73-.67=.06)$ was not supported by a chi-square test; only 10 subjects made a greater number of correct judg-

Table 1

Proportions of Correct Event-Frequency Judgments for Single and Paired Words of High Linguistic Frequency (hiLF) or Low Linguistic Frequency (loLF)

\begin{tabular}{lcccccc}
\hline & \multicolumn{6}{c}{ Word Frequencies } \\
\cline { 2 - 5 } Word Format & 0 & 1 & 2 & 3 & 4 & Total \\
\hline \multirow{5}{*}{ Single } & 1.00 & .79 & .79 & .29 & .21 & .53 \\
Paired & 1.00 & .83 & .63 & .58 & .29 & .67 \\
& \multicolumn{5}{c}{ loLF Words } \\
Single & .96 & .75 & .67 & .38 & .25 & .67 \\
Paired & 1.00 & .96 & .67 & .38 & .50 & .73 \\
\hline
\end{tabular}

Table 2

Mean Event-Frequency Judgments for Single and Paired Words of High Linguistic Frequency (hiLF) or Low Linguistic Frequency (loLF)

\begin{tabular}{lcccccc}
\hline & \multicolumn{6}{c}{ Word Frequencies } \\
\cline { 2 - 5 } Word Format & 0 & 1 & 2 & 3 & 4 & Total \\
\hline \multirow{5}{*}{ Single } & 0.00 & .96 & 2.54 & 3.63 & 4.17 & 2.24 \\
Paired & 0.00 & 1.13 & 2.00 & 3.00 & 3.58 & 1.94 \\
& \multicolumn{5}{c}{ loLF Words } \\
Single & .04 & 1.29 & 2.04 & 3.63 & 3.96 & 2.19 \\
Paired & 0.00 & 1.04 & 2.50 & 4.04 & 4.71 & 2.46 \\
\hline
\end{tabular}

ments for loLF words, compared with 7 who showed the opposite tendency $\left[\chi^{2}(1)=.38, p>.05\right]$.

Neither of the apparent advantages of paired over single words was supported by a chi-square test. The number of subjects making more correct EF judgments for paired than for single loLF words was 14, compared with 6 showing the opposite tendency $\left[\chi^{2}(1)=2.67, p>.05\right]$. The corresponding proportion for hiLF words was 10:7 $\left[\chi^{2}(1)=.38, p>.05\right]$.

Although these data have been presented according to EF categories to provide full information, that variable was not analyzed because there was no control for item specificity.

The correct-response data were also subjected to a more powerful analysis of variance. Mean correct EF judgments were 1.67 and 2.23 for single and paired hiLF words respectively, and 2.25 and 2.33 for the corresponding loLF means. Although neither main effect was reliable [LF: $F(1,22)=2.86, M S e=2.042, p>.05$; word format: $F(1,22)=2.85, M S e=3.375, p>.05]$, the interaction of LF and word format, suggested in the means listed above and in the overall proportions shown in Table 1, was reliable $[F(1,22)=4.15, M S \mathrm{e}=2.01, p$ $=.05]$.

The mean EF estimates for both sets of LF words are shown in Table 2 . There was obviously no difference in the single-word results, but there was a tendency for the loLF words to elicit relatively more higher frequency estimates in the paired-words format. This suggested difference was supported by the fact that 16 of the 24 subjects made higher paired loLF estimates, compared with 2 subjects who made higher paired hiLF estimates $\left[\chi^{2}(1)=\right.$ $8.17, p<.05]$. The corresponding figures for singleword presentations were 11 and $7\left[\chi^{2}(1)=.66, p>.05\right]$.

Neither of the differences between single and paired words was reliable by the chi-square analyses. There were 13 subjects who gave higher mean event-frequency judgments for single hiLF words, compared with 9 who showed the opposite tendency $\left[\chi^{2}(1)=.67, p>.05\right]$. For the loLF words, 14 subjects gave higher mean judgments for paired words, compared with 6 giving judgments in the opposite direction $\left[\chi^{2}(1)=2.67, p>.05\right]$.

No reliable main effects or interactions were found in the ANOVA of the estimate-size data. 


\section{DISCUSSION}

These results indicate a complex set of relationships between the linguistic frequency and the single-paired presentation variables, considered as determinants of event-frequency judgments. The effectiveness of the two variables apparently depends upon the particular measure used. When accuracy of judgment is measured, the number of occurrences of the loLF words was reliably better estimated, but only under the single-word presentation condition. When strength of the EF judgment is measured, the loLF words were estimated to be reliably more frequent, but only under the paired-words presentation condition.

The reliably higher apparent strength of the paired loLF words can hardly be used to explain the relatively greater accuracy of these same words, because the two results were obtained under different presentation conditions. Nevertheless, it might be noted that under appropriate circumstances, the increased strength factor could account for improved accuracy, at least for the higher event frequencies, in light of the commonly observed undershooting tendency for those items.

The reliably greater accuracy of EF judgments on singly presented loLF words than on singly presented hiLF words confirms the results of two experiments reported by Rao (1983). As Rao noted, the results are also consistent with the previously reported superior recognition of loLF words compared with hiLF words. In the present experiment, this difference cannot be attributed simply to increased size of judgments, because there was no consistent difference in that measure between hiLF and loLF words presented singly (Table 2 ).

The opposite results that were found for the paired-words presentations-no difference in accuracy as measured by correct judgments but a reliably higher level of estimates for the loLF words-also pose some interesting questions. The juxtaposition of hiLF and loLF words in the paired-words format seems not only to have increased the size of the judgments for the loLF words but also to have decreased the size of the judgments for the hiLF words. It may be hypothesized that loLF words tend to be more distinctive because of their relative rarity, and so are both better recognized and more accurately estimated for frequency when presented singly. But why, then, were they apparently overestimated in frequency when paired with more common words? Furthermore, why should frequency judgments of hiLF words be correspondingly depressed after pairing but not after single presentation? It is as though the distinctiveness of the loLF words was somehow accentuated by the pairing with hiLF words, and vice versa. An interpretation in terms of both contrast and strength concepts is indicated.

The superior EF judgments for single loLF words, which are normally better recognized but not better recalled when presented in homogeneous lists, cannot be readily accounted for on the basis of the complex results of recognition and recall studies, as reviewed in the introduction. Superior recall of multiple memory traces would be expected for the paired loLF words, on the basis of the results from several recall experiments, but apparently the contrast provided by the pairing served, in this case, mainly to increase the size of the frequency estimates.
Some comments are in order concerning the retrospective reports that were obtained from these subjects immediately after completion of the experiment (Marx, 1985). The subjects were asked to comment on how they had gone about making the unexpected EF judgments. In their reports, the procedure that was predominantly mentioned was some form of counting of multiple memory traces. As suggested above, the fact that the loLF words when paired with hiLF words produced reliably larger numerical estimates is quite consistent with such presumably improved retrieval of multiple memory traces. However, it is curious that in this experiment the accompanying accuracy of EF judgment that might also be expected did not occur. On the contrary, accuracy of EF judgment, at least as measured by correct judgments, was reliably better for loLF words that were singly presented. The contrast that was presumably produced by the mix of loLF and hiLF words within pairings apparently led to overretrieval of multiple traces, at the expense of accuracy. The fact that pairing also produced some reduction in the size of hiLF estimates should be noted. Because only the single hiLF words suffered in accuracy, however, there is no suggestion that this tendency towards reduction in estimates, and presumably in retrieved multiple memory traces, was detrimental to accuracy.

\section{REFERENCES}

Duncan, C. P. (1974). Retrieval of low-frequency words from mixed lists. Bulletin of the Psychonomic Society, 4, 137-138.

HARRIS, G., BeGG, I., \& MitTERER, J. (1980). On the relation between frequency estimates and recognition memory. Memory \& Cognition, 8, 99-104.

MARX, M. H. (1985). Retrospective reports on frequency judgments. Bulletin of the Psychonomic Society, 23, 309-310.

MaY, R. B., Cuddy, L. J., \& NorTon, J. M. (1979). Temporal contrast and the word frequency effect. Canadian Journal of Psychology, 33, 141-147.

MAY, R. B., \& TRYK, H. E. (1970). Word sequence, word frequency, and free recall. Canadian Journal of Psychology, 24, 299-304.

McCormack, P. D., \& Swenson, A. L. (1972). Recognition memory for common and rare words. Journal of Experimental Psychology, 95, 72-77.

RAO, K. V. (1983). Word frequency effect in situational frequency estimation. Journal of Experimental Psychology: Learning, Memory, \& Cognition, 9, 73-81.

SumbY, W. H. (1963). Word frequency and the serial position effect. Journal of Verbal Learning \& Verbal Behavior, 1, 443-450.

THORNDIKE, E. L., \& LORGE, I. (1944). The teacher's word book of 30,000 words. New York: Teachers College Press, Columbia University.

(Manuscript received for publication March 10, 1986.) 